\title{
Skid resistance demands of asphalt pavement during the braking process of autonomous vehicles
}

\author{
Zheng Binshuang ${ }^{1}$, Chen Jiaying ${ }^{1}$, Zhao Runmin $^{1}$ and Huang Xiaoming ${ }^{1, *}$ \\ ${ }^{1}$ School of Transportation, Southeast University, Nanjing 210096, China
}

\begin{abstract}
As the main operationality of AVs, the braking property is directly related to traffic safety. Major traffic accidents are often related to the braking distance, the side slip and hydroplaning during the emergency braking, which depends on the pavement skid resistance. Therefore, the estimation to relate AVs braking distance requirements with pavement peak friction coefficient to ensure a safe driving condition on expressway is of high practical significance. In this paper, the effect of AVs on braking performance parameters and dynamic friction on tire-pavement interaction are investigated. Based on the field test of the Coastal highway in Jiangsu province of China, this paper proposes an algorithm to determine time-dependent braking distance of AVs considering pavement frictional properties. According to the algorithm, an AVs braking system is provided to reach the maximum braking force for improving the AVs traffic safety. Furthermore, it revises the braking distance formula of Design Specification for Highway Alignment and the skid resistance threshold adopted by Technical Specifications for Maintenance of Highway Asphalt Pavement.
\end{abstract}

\section{Introduction}

Autonomous (or self-driving, driverless or robotic) vehicles are the kind of vehicles that do not require human factor input to be driven. According to reliable statistics, AVs approximately reduce vehicle crashes by $90 \%$ [1]. Autonomous (or self-driving, driverless or robotic) vehicles are the kind of vehicles that do not require human factors input to be driven. Being different from conventional vehicles, the $\mathrm{AV}$ is able to obtain more precise driving-conditions unlike the delay observed in human reaction time or the negative effects of human errors [2].

In recent years, traffic accidents of AVs mainly made by Google, Tesla as well as Uber began to occur constantly. There were eighteen traffic accidents on Google AVs during the past six years. As the main operationality of the AVs, the braking property is directly related to traffic safety. Major traffic accidents are often related to the braking distance, the side slip and hydroplaning during the emergency braking, which depends on the pavement skid resistance [3-5]. With the promotion of autonomous technology, sooner or later, the AVs will affect the geometric design of highways, transportation facilities and especially pavement performances such as skid resistance on rainy days. Therefore, the relationship between the AV-safe driving and the possible impacts on the skid resistance of expressway pavement need to be analyzed. The skid resistance of the pavement surface is primarily dominated by the surface frictional properties [6]. Pavement frictional properties decrease with the increase of road age. The maintained skid resistance depends on the pavement dynamic peak friction coefficient. Therefore, the estimation to relate AVs braking distance requirements with pavement peak friction coefficient to ensure a safe driving condition on expressway is of high practical significance.

In this study, the effect of $\mathrm{AVs}$ on braking performance parameters and dynamic friction on tirepavement interaction are investigated. Based on the field test of the Coastal highway in Jiangsu province of China, this paper proposes an algorithm to determine timedependent braking distance of AVs considering pavement frictional properties. According to the algorithm, an AVs braking system is provided to reach the maximum braking force for improving the AVs traffic safety. Furthermore, it revises the braking distance formula of Design Specification for Highway Alignment and the skid resistance threshold adopted by Technical Specifications for Maintenance of Highway Asphalt Pavement.

\section{Background: braking performance of AVs}

\subsection{Impact factors on braking distance}

Because of the detection range of the sensor, the maximum velocity of AVs is limited. The speed of AVs should be sufficiently low so that it can react to any event outside the sensor range. Frankly, the driver's reaction time of conventional vehicles will reduce as it becomes sensing time (in Figure 1). Braking time

\footnotetext{
* Corresponding author: zhengbs@seu.edu.cn
} 
is related to the AVs performance, and the time will reduce in the formula as follows:

$$
S_{\mathrm{T}}=S_{1}+S_{2}=\frac{v \cdot t}{3.6}+\frac{v^{2}}{254(\varphi+\psi)}
$$

Where, $S$ is the stopping sensing distance (or stopping distance) in $\mathrm{m} ; S_{1}$ is the vehicle reaction distance in $\mathrm{m} ; S_{2}$ is the vehicle braking distance in $\mathrm{m} ; v$ is the vehicle speed in $\mathrm{km} / \mathrm{h} ; t$ is braking time from hazard detected to the vehicle automatic stop. $\varphi$ is the adhesion coefficient between road surface and tire; $\psi$ is the road drag coefficient.

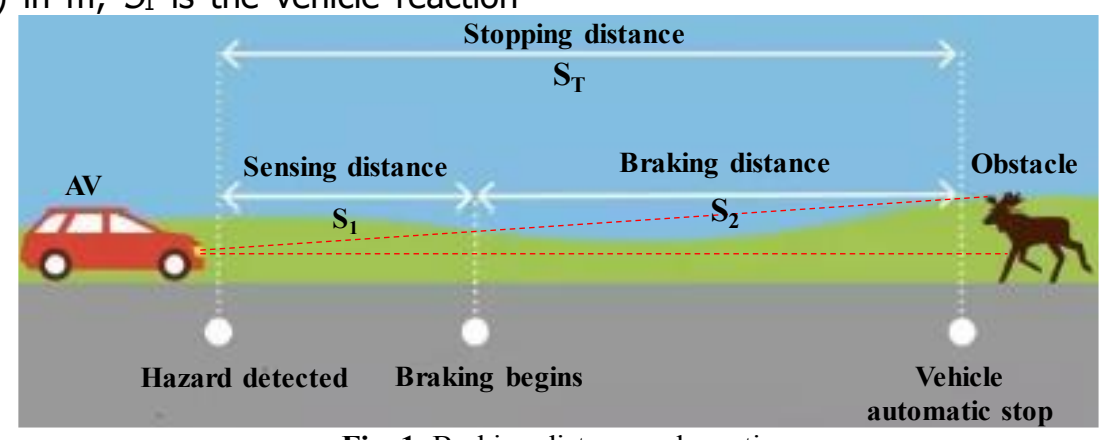

Fig. 1. Braking distance schematic

It can be observed from equation (1), the main impact of AVs on road horizontal geometric design is road surface frictional properties. Another, the influence of internal sensing device and the autonomous vehicle's performance should not be neglected yet. Therefore, providing a safe stopping distance is an important requirement in highway geometric design, and the braking distance is a major component. According to the theoretical analysis of road geometric design, the $A V s$ as vehicles are perceived to be higher speed, less intervehicle spacing and narrower lane width. In addition, the sensing reaction time (SRT) for AVs may be eliminated or reduced to milliseconds. Therefore, the stopping distance may be almost determined by the braking distance. In terms of road performance, the braking performance of an AV mainly depends on some factors, such as vehicle types, route alignment and special weather.

\subsubsection{Vehicle types}

Under good road conditions (the adhesion coefficient of tire-pavement is taken as 0.70 or larger), the braking distance is the shortest distance that the car brakes to the initial speed of $100 \mathrm{~km} / \mathrm{h}$. The braking distance for a typical vehicle is empirically ruled in Table 1 [7]. Whereas, it neglects the adhesion coefficient below 0.70 , which may impact the AVs' braking distance greatly. As for AVs, it should consider low frictional pavement condition that may cause a serious security problem because of sensor defect. To be well known, the commonly used sensing systems cannot measure the actual conditions of the road surface, such as water film, snow layer and particulate pollutants. These road surface coverings will greatly affect the road surface anti-sliding performance.

Table 1. Braking distance under different vehicle type

\begin{tabular}{|c|c|c|}
\hline $\begin{array}{c}\text { Vehicle speed } \\
(\mathrm{km} / \mathrm{h})\end{array}$ & Vehicle type & $\begin{array}{c}\text { Braking distance } \\
(\mathrm{m})\end{array}$ \\
\hline \multirow{4}{*}{100} & Jetta & 48.8 \\
\cline { 2 - 3 } & Buick GL8 & 45.8 \\
\cline { 2 - 3 } & Santana 2000 & 45.0 \\
\cline { 2 - 3 } & Passat & 43.9 \\
\cline { 2 - 3 } & Audi A6 1.8T & 42.3 \\
\cline { 2 - 3 } & Bora 1.8T & 40.0 \\
\cline { 2 - 3 } & BMW 745i & 37.1 \\
\hline
\end{tabular}

\subsubsection{Route alignment}

As mentioned above, the emergence of AVs will narrower lane width. Moreover, it will not consider the blind spot of the driver's sight for AVs' passenger in the route design. And, the lane width adjustment will reduce the length of the transition curve. Meanwhile, the reduction in the super-elevation runoff will result in the tangent runoff decreasing. From a straight segment to a curved segment, the transition curve will require better skid resistance to ensure the lateral safety factor of the driverless car.

During the process of uphill or downhill, the sensing range is limited due to the terrain, and it is impossible to timely sense the road surface condition in front. AVs need the road surface to have sufficient skid resistance force to maintain the vehicle braking stability as in an emergency. Considering the bottleneck and applicability of the development of driverless technology, this paper proposes an approach to regularly test the road surface skid resistance evaluation indicators to provide road performance data for the intelligent road management department.

When driving at an intersection, the AV needs to consider the distance between the front and the rear 
vehicle and the safety of the surrounding pedestrians under emergency braking condition. Only when driving overtaking, it needs to consider the lateral force coefficient, which is related to the road surface slip resistance. Under normal circumstances, the internal sensing system can safely brake in real time and is superior to traditional vehicles.

\subsubsection{Special weather}

In special weather, such as rainy days, snow, fog and sandstorms et al., the SRT of AVs will be greatly affected, especially the perception system will lose its effect. Therefore, the skid resistance performance of the road is crucial. The braking strategy of AV is timely implemented by the road performance indicators provided by the road management department. In short, avoiding traffic accidents as much as possible is the core purpose of the driverless technology.

In the future intelligent traffic management system, the intelligent maintenance of road skid performance will be a key issue for road researchers.

\subsection{Existing design specification of braking distance}

As for calculating braking distance, the road surface friction coefficient is often selected as a constant value without specifically considering the pavement frictional properties. For example, in Chinese Design Specification for Highway Alignment [8], the determination of braking distance is as follows:

$$
D=0.039 \frac{v^{2}}{g \cdot f}
$$

Where, $D$ is the braking distance in $\mathrm{m} ; v$ is the vehicle speed in $\mathrm{km} / \mathrm{h} ; f$ is the pavement coefficient friction depending on braking initial speed, load and pavement condition; $g$ is the vehicle the acceleration due to gravity in $\mathrm{m} / \mathrm{s}^{2}$ (taken as $\left.9.8 \mathrm{~m} / \mathrm{s}^{2}\right)$.

In addition, the Specification provided the braking distance under different vehicle speed using the formula above for the passenger car under wet condition, as shown in Table 2 [9]. Obviously, the braking distance varies with AVs operating conditions related to tire, pavement surface, water film on road surface et al.

Table 2. Braking distance under different road conditions

\begin{tabular}{|c|c|c|c|c|c|c|}
\hline $\begin{array}{c}\text { Design speed } \\
(\mathrm{km} / \mathrm{h})\end{array}$ & $\begin{array}{l}\text { Driving speed } \\
\quad(\mathrm{km} / \mathrm{h})\end{array}$ & $\begin{array}{c}f \\
\text { (Wet } \\
\text { condition) }\end{array}$ & $D(\mathrm{~m})$ & $\begin{array}{l}\text { Road condition } \\
(v=65 \mathrm{~km} / \mathrm{h})\end{array}$ & $\varphi_{\mathrm{p}}$ & $D_{p}(\mathrm{~m})$ \\
\hline 120 & 102 & 0.29 & 142.8 & Asphalt/concrete & $0.8 \sim 0.9$ & $21.0 \sim 18.7$ \\
\hline 100 & 85 & 0.30 & 95.8 & Wet asphalt & $0.5 \sim 0.7$ & $33.6 \sim 24.0$ \\
\hline 80 & 68 & 0.31 & 59.4 & Wet concrete & 0.7 & 24.0 \\
\hline 60 & 54 & 0.33 & 35.2 & Gravel & 0.6 & 28.0 \\
\hline 40 & 36 & 0.38 & 13.6 & Dirt road & $0.68 \sim 0.55$ & $24.7 \sim 30.6$ \\
\hline 30 & 30 & 0.44 & 8.1 & Snow & 0.2 & 84.1 \\
\hline 20 & 20 & 0.44 & 3.6 & Ice cover & 0.1 & 168.1 \\
\hline
\end{tabular}

Note: $\varphi_{\mathrm{p}}$ the peak adhesion coefficient of tire-road interface.

\section{Experimental program}

\subsection{Field Test}

The Coastal Highway in Jiangsu Province has been open to traffic for more than 10 years. The coastal expressway adopts a variety of pavement structure combinations. The upper layer is mainly composed of stone mastic asphalt (SMA-13), asphalt concrete (AC-13) and porous asphalt concrete (PAC-13) mixed materials.

With the increase of the service life, the skid resistance performance of some road sections has been significantly reduced under the comprehensive long-term effects of vehicle load and environment. In view of above situation, field test for skid resistance performance was conducted. According to the skid resistance performance of the expressway, the influencing factors and basic causes of skid resistance performance were analyzed. Then, the development law of skid resistance performance of the expressway was analyzed based on the historical maintenance history. The field test was planned along the different test sections. The evaluation of skid resistance adopted Technical Specifications for Maintenance of Highway Asphalt Pavement, that is, using the skid resistance coefficient as an evaluation index. In this study, the friction coefficient was extracted at different test speed. During the process, the surface temperature of all test positions is at $20{ }^{\circ} \mathrm{C}$. In the same test condition, the greater SFC value means higher skid resistance. 


\subsection{Data collection and analysis}

According to the field test data, the mean value of SFC in the second lane of the Coastal Highway is 52 at Lian-Tong direction, and at the Tong-Lian direction is 53. In the third lane, the mean value of SFC is 50 at Lian-Tong direction, and at the Tong-Lian direction is 49 . Overall, the road has good skid resistance performance. The third lane has a low level of skid resistance, which is directly related to its large traffic volume. Three influence factors on skid resistance of the expressway were considered in this study:

- Lane position at different traffic volume,

- Time-dependent attenuation and,

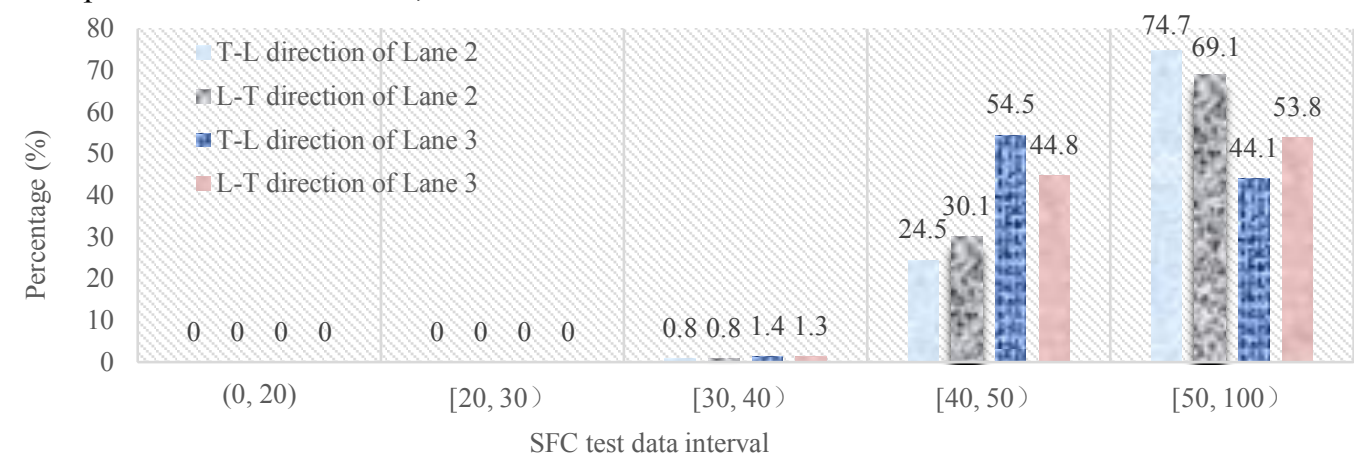

Fig. 2. Interval statistics of SFC test data for lane 2 and lane 3.

According to the historical data of maintenance, the SFC development law for the skid resistance insufficient evaluation unit of the second lane was analyzed yearly.

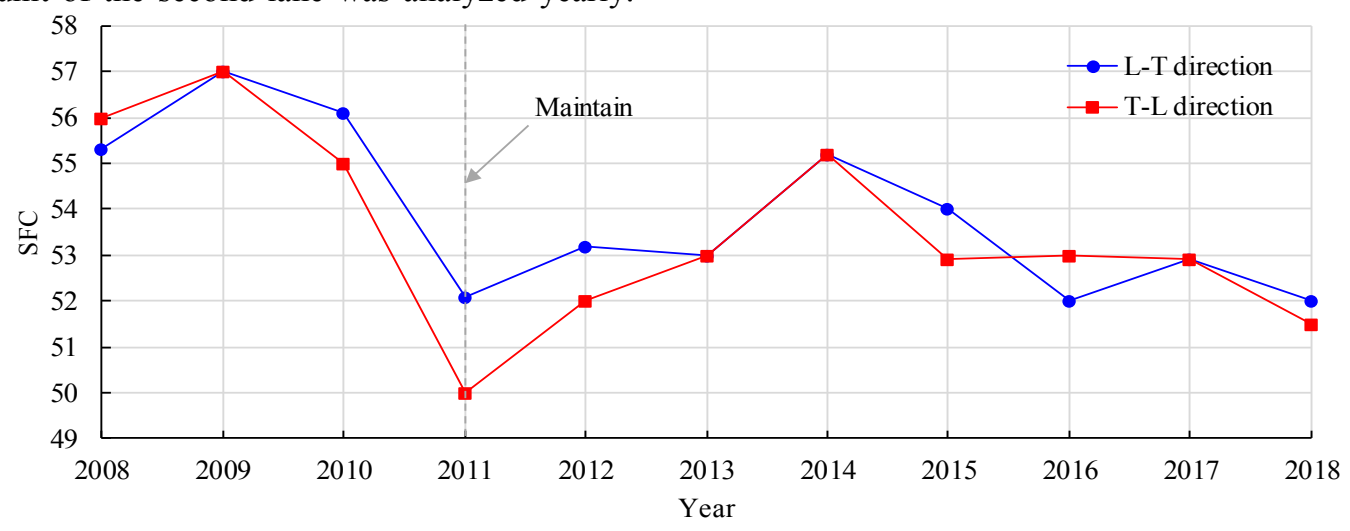

Fig. 3. Attenuation Trend of Pavement FSC On Second Lane
The SFC attenuation trend of the highway pavement second lane from 2008 to 2018 was drawn in Figure 3. 


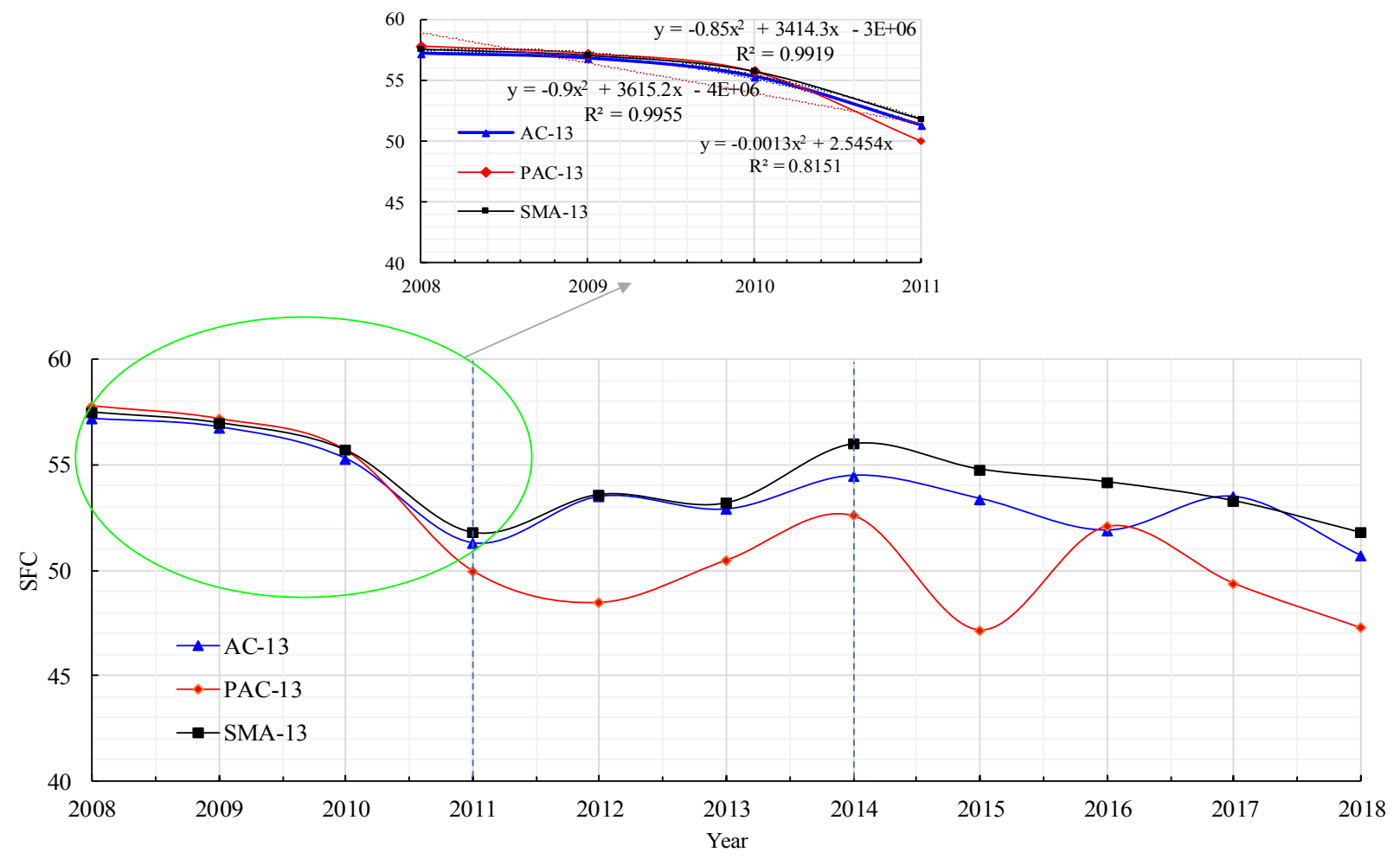

Fig. 4. FSC variation with different pavement structure

As shown in Figure 3, the skid resistance performance at the second lane is attenuated rapidly. After the special maintenance in 2011, the pavement skid resistance performance has been improved significantly. However, the skid resistance performance of the road surface has decreased again since 2014. This is caused by the rapid attenuation of the surface macro texture on the part road segment.

About different structure combinations of pavement, the variation of road skid resistance performance with time was analyzed (see Figure 4). It can be seen that the SMA-13 pavement has better skid resistance than AC-13 pavement, and the PAC-13 pavement is the worst under the effect of traffic load and environment. It is recommended to use SMA-13 pavement preferably in the future intelligent road pavement from the perspective of cost and management.

The regression analysis with the least square method is adapted to get the relationship between the SFC of road surface and time during the attenuation period of SFC. Founding that they have a good correlation as the equation (3),

$$
\begin{gathered}
\mathrm{SFC}=-0.85 t^{2}+3414 t-3 \times 10^{6} \quad, R^{2}=0.9919 \\
\varphi(t)=0.01(S F C)
\end{gathered}
$$

Where, $\varphi(t)$ is the frictional coefficient of tire-pavement interface; $t$ is the time, year.

Obviously, the skid resistance performance of pavement is time dependent and has peak value. By regularly monitoring the dynamic friction coefficient of the road surface, it is meaningful to provide a braking strategy for the AV to safely pass through the road segment with insufficient skid resistance performance. In a certain sense, it indirectly enables AVs to realize realtime identification of road surface friction characteristics.

Considering vehicle speed and tire-road friction coefficient changed with time, Fwa [4] proposed the speed-friction coefficient-time equation as vehicle brakes,

$$
\begin{gathered}
v(t)=v_{0}-\int_{0}^{T}[\varphi(t)+G] g d t \\
D=\int_{0}^{T} v(t) d t=\int_{0}^{T}\left[v(t=0)-\int_{0}^{t_{t}} a(t) d t\right] d t \\
=v_{0} t-\frac{1}{2} G g t^{2}-\int_{0}^{T}\left[\int_{0}^{t^{t}} \varphi(t) g d t\right] d t
\end{gathered}
$$

Where, $v(t)$ is the vehicle speed at any time, $t \in[0, T] ; v_{0}$ is the initial speed of vehicle in $\mathrm{km} / \mathrm{h} ; T$ is The time the vehicle begins to brake until it stops completely in s; $G$ is the road grade, positive value for uphill and negative value for downhill; $\varphi(t)$ is the friction coefficient of tireroad interface at any time; $g$ is the local gravity acceleration of the tire in $\mathrm{m} / \mathrm{s}^{2}$.

Based on the equation (3), equation (4) and equation (6), the braking distance as a function of time and friction coefficient is obtained as the prediction model for braking requirement when AVs driving on service highway.

\section{AV braking system}

According to the vehicle's braking performance, the ground braking force $F_{\mathrm{b}}$ is ultimately limited by the ground adhesion conditions [10]. The $F_{\mathrm{b}}$ meets with following Equation:

$$
F_{\mathrm{b}} \leq F_{\varphi}=F_{z} \cdot \varphi_{p}
$$

Where, $F_{\mathrm{b}}$ is the ground braking force in $\mathrm{N} ; F_{\varphi}$ is the adhesion of tire-pavement interface in $\mathrm{N} ; F_{Z}$ is the vertical 
load that the vehicle act on the ground in $\mathrm{N} ; \varphi_{\mathrm{p}}$ is the peak adhesion coefficient.

Therefore, the maximum deceleration $a_{\mathrm{bmax}}$ that the road can provide is equal to $\mathrm{g} \varphi_{\mathrm{p}}$. Thus, the road surface peak adhesion coefficient is predicted as the pressure threshold of the AVs' braking system, which can not only prevent the wheel from being locked, but also can greatly increase the braking force and shorten the braking distance. Based on the above analysis, the braking system for AVs was proposed, as shown in Figure 5.

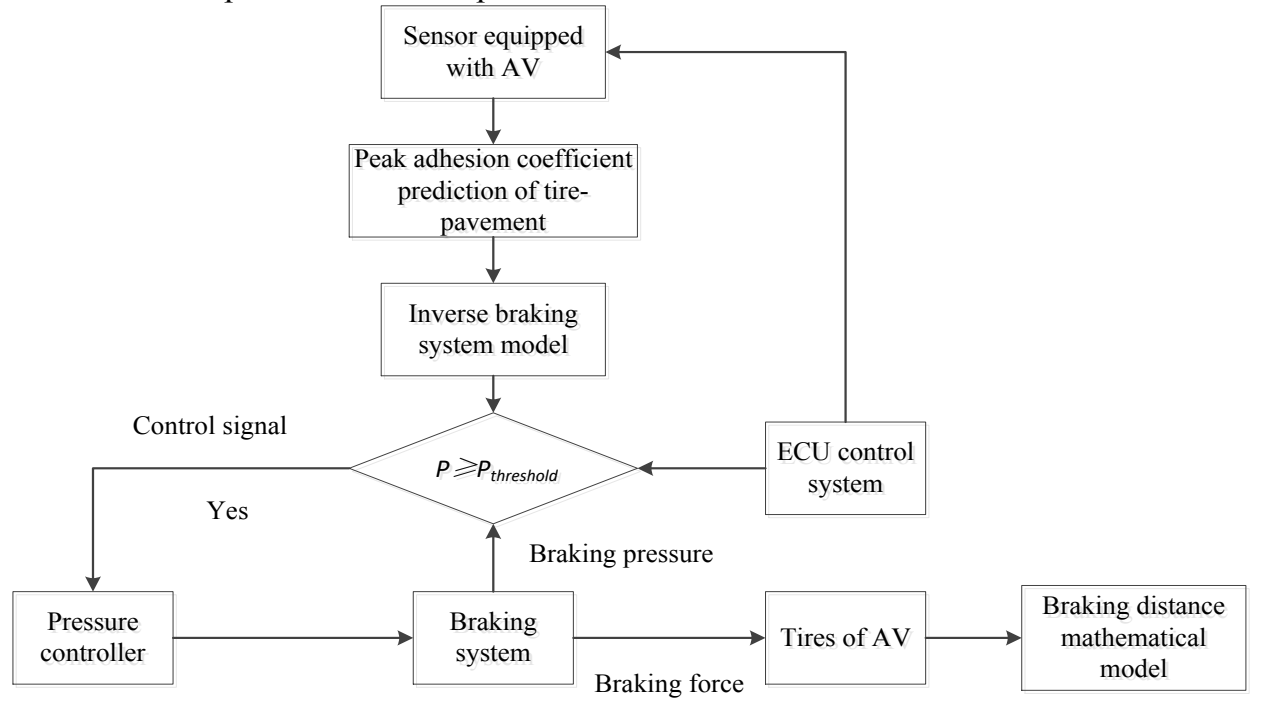

Fig. 5. AV braking system based on pavement frictional characteristic

According to the proposed AV braking system based on pavement frictional characteristic (see in Figure 5), The AV obtains the vehicle dynamics model response through the sensor. Combined with the above braking force mathematical model and the predicted road surface peak adhesion coefficient, the vehicle inverse longitudinal dynamics model is converted into the braking pressure threshold. Then, the electronic control unit (ECU) system equipped inside the vehicle calculates the required braking pressure through the braking pedal simulator. And the braking execution system responds quickly and outputs the maximum braking force to the tire to realize the optimal braking strategy for the driverless vehicle.

For emergency braking, the ECU determines whether the required braking pressure is greater than the pressure threshold. If it is greater than the pressure threshold, the braking cylinder pressure is controlled to be equal to the pressure threshold, and the maximum braking force with anti-lock braking based on the road surface friction characteristic is realized.

\section{Conclusions}

The purpose of this study was to study the braking performance of AVs and skid resistance of asphalt requirement. In this paper, the effect factors on braking performance of AVs and dynamic friction on tirepavement interaction were investigated. Field test for skid resistance performance was conducted. According to the skid resistance performance of the expressway, the influencing factors and basic causes of skid resistance performance were analyzed. Then, the development law of skid resistance performance of the expressway was studied based on the historical maintenance. The major findings of this study are:
(1) For the pavement frictional properties, the braking distance formula of Design Specification for Highway Alignment was revised.

(2) Considering vehicle speed and tire-road peak friction coefficient changed with time, the braking distance mathematical model was deduced.

(3) Based on the braking distance mathematical model, AV braking system based on pavement frictional characteristic was proposed, the maximum braking force with anti-lock braking based on the road surface friction characteristic can be realized.

\section{Acknowledgements}

The study is financially supported by National Natural Science Foundation of China (No.51778139) and the Research and Innovation Foundation for Graduate Students in Jiangsu Province of China (No. KYCX18_0146).

\section{References}

1. National Highway Traffic Safety Administration. National Motor Vehicle Crash Causation Survey: Report to Congress. National Highway Traffic Safety Administration, Washington, D.C., (2008).

2. Ye, L., T. and Yamamoto. Modeling Connected and Autonomous Vehicles in Heterogeneous Traffic Flow. Physica A Statistical Mechanics \& Its Applications, 490, 269-277 (2018).

3. Ong, G. P., T. F. Fwa. A Mechanistic Interpretation of Braking Distance Specifications and Pavement Friction Requirements. Transportation Research Record, Journal of the Transportation Research Board, 2155, 145-157, (2010). 
4. Chu, L. J., T. F. Fwa. Pavement Skid Resistance Consideration in Rain-related Wet-weather Speed Limits Determination. Road Materials \& Pavement Design, 1-19, (2016).

5. Kogbara, R. B., E. A. Masad, and E. Kassem. A stateof-the-art review of parameters influencing measurement and modeling of skid resistance of asphalt pavements. Construction \& Building Materials. 114, 602-617, (2016).

6. Mccarthy, R., G. W. Flintsch, and S. W. Katicha. New Approach for Managing Pavement Friction and Reducing Road Crashes. Transportation Research Record Journal of the Transportation Research Board, 2591, 23-32, (2016).

7. Yu, Z. S. Automotive Theory. China Machine Press, Beijing, (2009).

8. Design Specification for Highway Alignment. Publication JTJ D20-2006. The People's Republic of China Ministry of Communications, (2006).

9. Cong, L. and X. Huang. Influence of Pavement Frictional Properties on Braking Distance. Journal of Southeast University (English Edition), 27(2), 210212, (2011).

10. Zhang, L. F. Research on Key Technology of Active Collision Avoidance Braking System Based on Pavement Identification. Jiangsu University, (2017). 